\title{
Neurologic improvement after thoracic, thoracolumbar, and lumbar spinal cord (conus medullaris) injuries
}

\author{
James S. Harrop, MD \\ Thomas Jefferson University Hospital \\ Swetha Naroji \\ Thomas Jefferson University Hospital \\ Mitchell G. Maltenfort, PhD \\ Thomas Jefferson University Hospital \\ John K. Ratliff, MD \\ Thomas Jefferson University Hospital \\ Follow this and additional works at: https://jdc.jefferson.edu/orthofp \\ ftavropoula L. Tjoumakaris \\ momas of therson University Hospitas \\ Let us know how access to this document benefits you
}

\section{See next page for additional authors}

Recommended Citation

Harrop, MD, James S.; Naroji, Swetha; Maltenfort, PhD, Mitchell G.; Ratliff, MD, John K.;

Tjoumakaris, Stavropoula L.; Frank, Brian; Anderson, M.D., D. Greg; Albert, MD, Todd; and

Vaccaro, MD, PhD, Alexander R., "Neurologic improvement after thoracic, thoracolumbar, and lumbar spinal cord (conus medullaris) injuries" (2011). Department of Orthopaedic Surgery

Faculty Papers. Paper 29.

https://jdc.jefferson.edu/orthofp/29

This Article is brought to you for free and open access by the Jefferson Digital Commons. The Jefferson Digital Commons is a service of Thomas Jefferson University's Center for Teaching and Learning (CTL). The Commons is a showcase for Jefferson books and journals, peer-reviewed scholarly publications, unique historical collections from the University archives, and teaching tools. The Jefferson Digital Commons allows researchers and interested readers anywhere in the world to learn about and keep up to date with Jefferson scholarship. This article has been accepted for inclusion in Department of Orthopaedic Surgery Faculty Papers by an authorized administrator of the Jefferson Digital Commons. For more information, please contact: JeffersonDigitalCommons@jefferson.edu. 


\section{Authors}

James S. Harrop, MD; Swetha Naroji; Mitchell G. Maltenfort, PhD; John K. Ratliff, MD; Stavropoula L.

Tjoumakaris; Brian Frank; D. Greg Anderson, M.D.; Todd Albert, MD; and Alexander R. Vaccaro, MD, PhD 


\title{
As submitted to:
}

\author{
Spine
}

\section{And later accepted as:}

\section{Neurologic improvement after thoracic, thoracolumbar, and lumbar spinal cord (conus medullaris) injuries}

\section{Volume 36, Issue 1, 1 January 2011, Pages 21-25 DOI: 10.1097/BRS.0b013e3181fd6b36}

\section{INTRODUCTION}

Traumatic spinal cord injuries (SCI) are catastrophic events that typically affect younger populations and often result in tremendous economic loss and societal burden. These injuries create severe physical limitations which depend on the neurologic level, severity of the injury, patient age and medical co-morbidities. Predicting neurologic recovery is a complex process; there is only sparse literature detailing neurologic recovery particularly after thoracic and lumbar spinal cord injuries. The likelihood and degree of neurologic recovery depends in part upon the anatomic region of the spinal cord injury. For example, (T1-T5) are more often complete with less potential for meaningful neurologic recovery believed to be due the tenuous blood supply in this region as well as the high degree mechanism needed to create an injury due to the stability of the chest wall and spinal column in the region..

In the thoracolumbar region of the spine, the clinical manifestations of a spinal cord injury are influenced by unique anatomy of the conus medullaris. The conus medullaris contains the anterior horn cells for the distal lumbar and sacral spinal cord 
segments and is surrounded by nerve roots for the upper lumbar segments. Relatively less volume of the cord in this region is taken up by the ascending and descending spinal cord tracts. Depending on the vertebra that is fractured, the neurologic injury can manifest a unique combination of upper and lower motor neuron injury along with bowel/bladder and lower extremity dysfunction. If the trauma is localized over the distal most spinal cord there may be a predominant component of lower motor neuron, rather than upper motor neuron, injury. Opportunity for neural recovery at this level potentially would be greater compared to the upper spinal cord (ie: thoracic), as lower motor or peripheral nerve injuries tend to have a better prognosis for neurologic recovery.(Figures 1 and 2) This tendency of the lower motor neurons at the site of a spinal cord injury to recover is referred to as "root escape."

Differentiating recovery rates based on anatomic regions for thoracic, thoracolumbar and lumbar spinal cord injuries has been suggested by some authors but has not been previously detailed. This article analyzes a large population of spinal cord injury patients and differentiates neurologic recovery by anatomic region of the cord injury to better define recovery potential for subsets of the spinal cord injured population.

\section{$\underline{\text { METHODS }}$}

Over a 10-year period between January 1995 to Jan 2005, 1746 consecutive spinal cord and spinal injured patients were seen, evaluated, and treated through the Delaware Valley Regional Spinal Cord Injury Center, a Level 1 trauma referral center. Patients who upon entry to the hospital were confirmed to have a spinal cord or column trauma were examined by a multimodality clinical team consisting of Trauma, Neurological, and 
Orthopedic Surgeons, and Physiatrists. Initial examinations on admission $(<24 \mathrm{hrs}$ from trauma), as well as sequential follow-up examinations were performed and recorded utilizing the ASIA grading system. Patient records were maintained in an IRB approved spinal cord injury database. Specific data points for gender, age, location of neurologic injury, functional grade, etiology of injury, and mortality rate were documented.

A retrospective analysis of the relationship between the anatomic location of injury (T4 to S5) and the degree of neurological recovery was performed under an additional and separate approval by the IRB committee. The thoracic region was defined as T4-9, the thoracolumbar region as T10-T12 and the lumbar region as (L1-S5). Patients were included in the analysis if they meet the inclusion criteria of a non penetrating traumatic spinal cord injury (ASIA A-D), age greater than 14 years, with complete initial and one-year ASIA examinations Excluded from analysis were peripheral nerve injuries (lumbar plexus) and cases where physical exams were not reliable due to concurrent injuries (ie: closed head injury).

\section{Statistical Analysis}

Chi-squared tests and logistic regression were performed using the JMP software package (version 7.02. SAS Institute, Cary NC).

\section{$\underline{\text { RESULTS }}$}

During the 10 year period of analysis, 282 patients were identified on the initial database query that met the inclusion criteria of a T4-S5 level traumatic spinal cord injury, consistent with an ASIA A through ASIA D neurological examination. Of these, $44 \%(124 / 282)$ were thoracic, $30 \%(85 / 282)$ were thoracolumbar, and $26 \%(73 / 282)$ were 
lumbar injuries. Following elimination of penetrating and non-traumatic injuries, a total of 150 cases remained. Full neurologic follow up data was available for 95 patients ((43\% thoracic, $27 \%$ thoracolumbar, $29 \%$ lumbar). As the pool of eligible subjects was refined, the distribution of injury levels remained fairly constant. The admission and 1year follow-up ASIA scores are tabulated and presented in Table 1. Only one patient worsened neurologically at one year follow up (T4-T9, B to A). There was no relationship between anatomic region and time to follow-up.

Lumbar patients (L1-S5) recovered at a rate of $89.3 \%$, versus $22.3 \%$ for Thoracic and Thoracolumbar patients $(\mathrm{T} 4-12)(\mathrm{p}<0.0001)$. Only $9.4 \%$ of ASIA A patients showed recovery, compared to $95.2 \%$ of ASIA D patients; ASIA B patients demonstrated a $62.5 \%$ recovery rate, while ASIA $\mathrm{C}$ had a $76.9 \%$ recovery rate $(\mathrm{p}<0.0001)$. When anatomic location and neurologic grade were considered jointly in a multivariate analysis, ASIA A and thoracic or thoracolumbar patients had only a $4.1 \%$ rate of recovery, versus $92 \%$ for lumbar region and incomplete patients (ASIA B-D) and $72 \%-75 \%$ for the rest of the patients.

\section{Transition at the level of injury}

A logistic regression analysis was performed to assess each anatomic region's potential for neurologic recovery. Each segment was assigned a consecutive integer ( $\mathrm{T} 4=1, \mathrm{~T} 5=2$, etc), and the estimated probability of recovery was fit to anatomical region.. Because logistic regression forces a smooth curve, sharp transitions between regions might have been lost. The fit $(\mathrm{p}<0.0001)$ suggested that in the upper thoracic region, the chance of recovery was $16.7 \%$ (6.9\% at $\mathrm{T} 4$ to $30.0 \%$ at $\mathrm{T} 9)$; in the 
thoracolumbar region, $46.6 \%$ (37.9\% at $\mathrm{T} 10$ to $55.3 \%$ at $\mathrm{T} 12)$; and in the lumbar region, $83 \%(63.7 \%$ at $\mathrm{L} 1$ to $95.4 \%$ at S3). (Figure 3$)$

\section{Demographic and Mechanism versus Recovery}

Age was not significantly related to recovery. The 40 patients who recovered had a mean age of 37.3 years with a standard deviation of 15.8 ; the other 54 patients had a mean age of 33.6 with a standard deviation of 12.0. Also, gender was not related to the odds of recovery: twenty percent of both patient populations were female. Ethnicity and mechanism of injury were both associated with neurorecovery. $57.9 \%$ of patients with falls recovered, versus $31.6 \%$ of patients incurring other mechanisms of injury $(\mathrm{p}<0.01) .47 .4 \%$ of white patients demonstrated signs of recovery, versus $17.7 \%$ of non-white patients $(\mathrm{p}<0.05)$. However, when both these factors were included in a multivariate analysis with anatomic region and ASIA grade, their individual contributions to neural recovery was not significant. With respect to patients with adequate follow-up, 76.4\% of non-white patients were ASIA A vs. 51.3\% of white patients $(\mathrm{p}<0.05)$. Among patients with falls as an injury mechanism, 26.3\% were thoracic injuries (T4-T9) while $42.1 \%$ were lumbar; among patients with other injuries, $54.4 \%$ were thoracic injuries while $21.1 \%$ were lumbar. This association between mechanism of injury and anatomic region of injury was significant to $\mathrm{p}<0.05$.

\section{$\underline{\text { DISCUSSION }}$}

Trauma patients with spine and spinal cord injuries are categorized based on the level of their bony and neurologic injury. $(4,11)$ Defining the level of the osseous vertebral column injury can occasionally be affected by anatomic variations in the 
skeletal segments, where it has been suggested $15-20 \%$ of patients have "altered" lumbosacral vertebrae anatomy which may lead to confusion of the bony injury level(14, 21) (23). In contrast, the neurologic level of injury is consistently defined by a detailed physical examination as standardized by the American Spinal Injury Association (ASIA) which localizes the injured neural tissue to the anatomic spinal cord segment.(5) The neurologic level is definable and can be followed over time to define neurologic recovery following an acute spinal cord injury.

The relationship between the bony vertebral column injury and the level of a spinal cord injury can vary depending on level of the vertebral column injury as discussed above. In addition, the length of the vertebral column and spinal cord can differ significantly. The distal tip of the spinal cord (conus medularis) most commonly terminates at the L1-2 level, but in some patients may end in the thoracic region or distally at the sacrum. $(13,14,25)$ For this reason, the neurologic or spinal cord injury level may vary significantly between patients with similar spinal column injuries.

Another anatomic variable effecting injuries to the cord is the length of each spinal cord segment. In the upper thoracic cord, the spinal cord segments such as T2 or T3 (neurologic not vertebral) are approximately 15-20 $\mathrm{mm}$ in length; this decreases to approximately $5 \mathrm{~mm}$ for the lumbar and sacral spinal cord.(12) (Figure 4)

For this study, the most superior level of motor function in the thoracic region was defined as T4 to eliminate the potential influence on neurologic level confusion due to cervical and upper thoracic spinal cord injuries. Due to the presence of a high percentage of lower motor neurons within the thoracolumbar junction, clinical SCI trials often exclude injuries below the T10 level.(6-9) In addition, studies have noted a 
significantly limited neurologic recovery potential with SCI proximal to T10.(27, 31)

For this study, we defined the thoracolumbar region (T10 to T12) as a transitional region due to the possible presence of both upper and lower motor neurons in the spinal cord.

In this series patients with thoracic region injuries had the lowest rate of neurologic recovery, only 9 of $41(22 \%)$ had any neurologic recovery. Further, the most severe injuries had the least recovery. Only $3 \%(1 / 03)$ of thoracic motor complete ASIA A patients had any improvement in neurologic function at one year follow-up. Waters et al noted a similar recovery rate in their prospective analysis of 148 patients with thoracic paraplegia. 142 remained as complete neurologic injuries at follow-up. Specifically, any patient with a level of injury above T9 did not regain any lower extremity function. (27, 31) However, the series of Waters et al was a heterogeneous SCI population consisting of penetrating and non-penetrating spinal cord injury patients. Rahimi-Movaghar et al further noted that despite surgical decompression, complete thoracic patients demonstrated no apparent neurologic recovery.(19)

The spinal cord segment named after their respective lumbar and sacral levels lies at a variable distance from the actual vertebral bony pedicle around which the nerve roots course and for which they are named.(12) This differs significantly from the cervical and proximal thoracic regions. The importance is that lower motor neurons have a much improved recovery rate even in complete (ASIA A) patients. This principle is illustrated in complete cervical ASIA A patients where the last functioning nerve roots are typically at the level immediately adjacent to a bony fracture or dislocation.(10) (22) This principle is also illustrated in the series of Waters et al, where $38 \%$ of the patients at or below T9 demonstrated some degree of recovery, and twenty percent of patients with a 
T12 or lower level of injury regained the ability to ambulate with devices, and 50\% (3 of 6) regained voluntary bowel and bladder function. $(27,31)$ In another series, thoracolumbar patients had notable improvement in bladder function (63.6\%) and nerve root recovery at follow up (83.3\%).(20)

The improved apparent recovery rates of the lumbar region of the spinal cord is most likely due to the greater proportion of lower to upper motor neurons in the distal spinal cord and the possibility for "root escape" in the lumbar region.(13) Seven of fifteen lumbar level patients were motor complete (4 ASIA A and 3 ASIAB) injuries and subsequently sustained recovery. Marino illustrated that this neurologic recovery after spinal cord injury is mediated through sprouting of the nerve roots using single fiber EMG studies (17). The potential of non-functioning nerve roots ( $0 / 5$ strength) to regain strength after motor complete cervical spinal cord injury has been well documented.(15, $16,32)$ This phenomenon has been labeled "root recovery" describing that lower motor neurons at the site of the injury which had no initial function can recover.

In the distal spinal cord or conus medullaris there is an increased potential for neurological recovery due to increased lower motor neuron ability to repair and due to root escape. (20) The "transition zone" segments, T10 to T12, demonstrated significantly less neurologic recovery than the lumbar spinal cord injured patients, with only 6 of 26 showing any significant recovery $(\mathrm{p}<0.0001)$. The hypothesis that the three anatomic regions are distinct neurologic zones with differences in recovery potential was tested by attempting to estimate the spinal level where the chance of recovery changed. The segment at which the partition was made was moved systematically (Figure 5), and chisquare statistics were calculated comparing recovery rates above and below the selected 
level. The chi-squared statistic, a measure of the departure of the recovery rates from random chance, was greatest when the division was at T12 \& higher vs. L1 and lower ( $22 \%$ chance of recovery vs. $89 \%$ chance of recovery, respectively). Using the prior logistic regression results (Figure 3), it was found that in the upper thoracic region, the chance of recovery averaged $16.7 \%$ (6.9\% at T4 to $30.0 \%$ at T9); in the thoracolumbar region, $46.6 \%$ (37.9\% at $\mathrm{T} 10$ to $55.3 \%$ at $\mathrm{T} 12)$; and in the lumbar region, $83 \%$ (63.7\% at L1 to $95.4 \%$ at S3), suggesting that the 3 zones behave differently with regards to neurologic recovery.

\section{CONCLUSION}

There are numerous obstacles in differentiating potential recovery rates of SCI patients. With approximately 10,000 new SCI patients in the United States on an annual basis, predicting patient outcomes is an important public health concern. With recent advances in the understanding of cellular biology and the biomolecular aspects of neural repair, ongoing clinical and translational trials are only beginning to appreciate of the complexity of spinal cord regeneration. Combining all regions of the spine in spinal cord injury trials may be misleading as the lumbar and sacral spinal cord regions recover at different rates than the thoracic or thoracolumbar cord. This point should be observed for future clinical trial design. 


\section{FIGURE LEGENDS}

Figure 1 represents thoracic spinal cord (1A). Note there is significant area between the exiting lower motor nerve roots and the adjacent level. When a spinal cord injury occurs over area in the red box the patient loses the lower motor neurons as well as distal motor neuron affected by descending motor tracts (1B). Patients typically regain the most proximal nerve root function (green) but have less to no return of distal motor function.(1C)

Figure 2 represents region of the conus medullaris or lumbar spinal cord (2A). Note compact area between the exiting lower motor nerve roots and the adjacent level (2B). When a spinal cord injury occurs over area in the red box the patient loses the lower motor neurons as well as distal motor neuron affected by descending motor tracts. A lumbar SCI typically regain the most proximal nerve root function (green) but have less to no return of distal function which represent bowel and bladder (2C).

Figure 3. Logistic regression fit of the probability of recovery to the level of spinal injury, assigning a number to each level. The fit is significant $(\mathrm{p}<0.0001)$. The odds ratio per level is 1.42 (95\% $1.24-1.68)$, suggesting the odds of recovery double for every two spinal levels lower.

Figure 4. Length of spinal cord segments according to neurological level (mm). The average length of spinal cord segments decreases after the midthoracic level as the spinal cord approaches the sacrum. This anatomic variability throughout the spinal cord is important to keep in mind when considering neurological recovery.

Figure 5. The spinal cord is divided into upper and lower regions, and as the transition shifts (upper region is marked on X-axis), the recovery rates (closed symbols) is plotted with the chi-squared statistic (open square) comparing the two rates. The chi-squared statistic was greatest when comparing T4-T12 to L1-S3 (22\% of recovery vs. $89 \%$ chance of recovery). 